Methods 1307 in-patients with ST segment elevation ACS from 64 hospitals across China were received different therapies in hospitals and a standard questionnaire was used to get information of the patients including demographic, treatments and in-hospital outcomes. We analysed the status of application of reperfusion and aspirin, ACEI, $\beta$-blocker, LWMH, Clopidogrel and cholesterol lowering agents on ST segment elevation ACS patients.

Results 1 . The were no significant differences in baseline characteristics between the patients from tertiary hospitals and that from the secondary hospitals. 2. $30.9 \%-69.4 \%$ received reperfusion therapies. $1.3 \%-62.7 \%$ received primary $\mathrm{PCI}, 1.9 \%-45.8 \%$ received Thrombolysis, and nearly $46.2 \%$ did not receive any form of reperfusion. Reperfusion therapy was more often used in tertiary hospitals $(48.2 \%)$ than in secondary hospitals $(6.46 \%)$. Thrombolysis was more often in secondary hospitals than that in tertiary hospitals (36.8\% vs $14.6 \%$ ) 3. Percentage of medications in ST ACS patients in different areas: aspirin (88.0\%-98). 6\%), ACEI (60.5\%84). $4 \%$ ), $\beta$-blocker (55.8\%-84.4\%), LWMH54.2\%-94.2\%), Clopidogrel (14.3-88.6), cholesterol lowering agents Statins (51.9\%$90.9 \%)$; 4. Major in-hospital events and death rates were significantly higher in secondary hospitals than in tertiary hospitals; 5. Mortality and congestive heart failure rate were significantly higher in patients with no reperfusion therapy compared to patients underwent reperfusion. The incidence of combined outcomes (death or MI, and death, MI or Stroke) was also higher in patients without reperfusion therapy 6. Multivariate logistic regression analysis showed that age $>75$ years, hypertension, diabetes, reperfusion, aspirin, $\beta$-blocker, ACEI/ARB inhibitor use were associated independently with in-hospital mortality.

Conclusion In the most tertiary hospitals in China the application of medications being proved effective by evidence-based medicine in clinical practice is better than that of the secondary hospitals, but there is a big gap between guidelines and current management of ST segment elevation ACS in China, and the application status in China could be further improved.

\section{E0259 CONTROL STATUS OF CHOLESTEROL OUTPATIENTS WITH HEART ARTERY DISEASE IN CHINA}

doi:10.1136/hrt.2010.208967.259

Zhang Huiying, Wang Jiansong, Guo Lai-Jing, Xing Liying. Chronic Disease Institute, Beijing University Shougang Hospital

Objectives To evaluate the current control status of cholesterol among outpatients with heart artery disease in China.

Methods Sixty-four hospitals across China, including 32 secondary hospitals and 32 tertiary hospitals were selected for baseline survey. Fifty outpatients diagnosed heart artery disease were recruited consecutively in each participated hospital. Information for 1806 patients was collected, and control status of cholesterol among the patients was analysed.

Results (1) Mean age of the patients was 65.10. Seventy point five percent of the patients were male and $29.5 \%$ were female. (2) Overall $26.2 \%$ attained the cholesterol goal in the 1806 patients of heart artery disease, the goal attainment rate of cholesterol among male $(30.5 \%)$ patients was higher than that among female $(15.8 \%)$ patients. (3) The goal attainment rate of cholesterol was highest in middle China (39.7\%) and lowest in northeast area (14.9\%) among 7 geographic district (north China, east China, south China, middle China, northeast, northwest, southeast) $(p<0.01)$.

Conclusions Only 26.2 heart artery disease patients attained the cholesterol goal, the rate varied significantly among sex, different areas and different degree hospitals. It was essential to pay more atttention on control of cholesterol for secondary prevention of cardiovascular disease. e0260 IMPACT OF PSYCHOLOGICAL INTERVENTION ON NEGATIVE EMOTION AND LIFE OUALITY OF PATIENTS WITH ACUTE CORONARY SYNDROME

doi:10.1136/hrt.2010.208967.260

Zhang-Qiang Chen, Lang Hong, Hong Wang, Qiu-Lin Yin, Lin-Xiang Lu. Jiangxl Provincial People's Hospital

Objective To observe the impact of psychological intervention on patients' negative emotion and life quality with acute coronary syndrome (ACS).

Methods 80 patients with liver cancer were chosen in our hospital from January 2007 to December 2009, and randomly divided into experimental group (40 cases) and control group (40 cases), the followup assessment of psychological status and life quality were performed by taking symptom self-assessment form (SCL-90), self-rating depression scale (SDS), Self-Rating Anxiety Scale (SAS), Quality of Life Scale (ARES-S), and the targeted psychological intervention relayed on the basis of the initial results of assessment and intervention results were analysed to assess the differences between the two groups.

Results The patients with liver cancer companied with early poor mental health, anxiety, depression, obviously, the life quality significantly decreased; the initial results of the assessment were SDS (56.4 \pm 8.3$)$ points, SAS $(55.6 \pm 5.2)$ points, SCL-90 (1.9 \pm 0.4$)$ points, CARES-SF $(25.2 \pm 15.5)$ points. 1 month after intervention, the psychological status and life quality of patients than in a month ago has significantly improved, the scale scores were SDS (42.1 17.5 ) points, SAS $(42.5 \pm 5.2)$ points, SCL-90 (1.4 \pm 0.3$)$ points, CARES-SF $(32.5 \pm 16.7)$ points, the scores were significantly difference $(p<0.01$ or $\mathrm{p}<0.05)$. between before and after psychological intervention. Therefore, the psychologically negative emotions (depression, anxiety, hostility, fear) in the test group after the intervention were significantly decreased (the first two $\mathrm{p}<0.01$, both of $\mathrm{p}<0.05$ ).

Conclusion Psychological intervention can effectively relief negative emotional and psychological stress and help to improve the life quality of the patients with acute coronary syndrome.

\section{e0261 RELATIONSHIP OF PHYSICAL EXERCISE AND CHEST PAIN RECURRENCE OF 2401 CORONARY ARTERY DISEASE (CAD) PATIENTS}

doi:10.1136/hrt.2010.208967.261

Zhang Huiying, Wang Jiansong, Guo Lai-jing, Xing Liying. Chronic Disease Institute, Beijing University Shougang Hospital

Objective To study the relationship of physical exercise and chest pain recurrence of $\mathrm{CAD}$, and if physical exercise could reduce rate of chest pain recurrence and improve quality of life.

Methods 64 hospitals across China mainland, involving 2401 patients, 50 out-patients with $\mathrm{CAD}$ in each hospital were included and standard questionnaires were used to get relative information on patients' demographic, physical exercise, chest pain recurrence et al. Results It existed significant differences between exercise and chest pain recurrence.

Conclusions Appropriate physical exercise could reduce rate of chest pain recurrence.

\section{$\mathrm{e} 0262$ ANALYSIS OF SMOKING STATUS AMONG RESIDENTS IN BEIJING SHOUGANG DISTRICT}

doi:10.1136/hrt.2010.208967.262

Zhang Huiying, Wang Jiansong, Dong Liguang, Xing Liying, Guo Lai-Jing. Chronic Disease Institute1, Beijing University Shougang Hospital

Objective This survey aims to know the current status of smoking among residents in Beijing Shougang district and analyse the relative factors. 